\title{
The VSOP Survey Program
}

\author{
J.E.J. Lovell \\ Australia Telescope National Facility, Epping, New South Wales 2122, \\ Australia \\ E.B. Fomalont, G.A. Moellenbrock \\ National Radio Astronomy Observatory, Charlottesville, VA, USA
}

S. Horiuchi, H. Hirabayashi

Institute of Space and Astronautical Science, Sagamihara, Kanagawa 229-8510

\author{
W.K. Scott \\ University of Calgary, Calgary, Alberta, T2N 1N4, Canada \\ R. Dodson \\ University of Tasmania, Hobart, Tasmania 7000, Australia
}

\begin{abstract}
The VSOP Survey Program contains 402 bright, small diameter extragalactic radio sources. Pre-launch observations indicated that 113 sources were probably undetectable on space-ground baselines, and so VSOP observations are being undertaken at $5 \mathrm{GHz}$ of the remaining 289 sources. Progress to date is described.
\end{abstract}

\section{Introduction}

VSOP (VLBI Space Observatory Programme) observations are made with the satellite HALCA and arrays of ground radio telescopes (Hirabayashi et al. 1998; 2000a). The majority of HALCA observations are made for peer-reviewed proposals as part of General Observing Time (GOT). A typical GOT observation uses HALCA with $\sim 10$ ground telescopes for a duration of $\sim 10$ hours. Highlights from GOT observations to date are reviewed elsewhere (Murata et al. 2001). VSOP Survey Program observations are made with HALCA and $\sim 3$ ground telescopes over $\sim 6$ hours. One of the main aims of the survey is to study the statistical properties of active galactic nuclei at sub-milliarcsec resolution, corresponding to brightness temperatures above $\sim 10^{12} \mathrm{~K}$ (Hirabayashi et al. 2000b).

The complete VSOP Survey sample includes all cataloged extragalactic radio sources in the sky with: a cataloged total flux density at $5 \mathrm{GHz}>0.95 \mathrm{Jy}$, a spectral index $\alpha>-0.45$ (where $S \propto \nu^{\alpha}$ ), and a galactic latitude $|b|>10^{\circ}$. In 
addition, 37 extragalactic sources with total flux densities $>5$ Jy were included regardless of their spectral index and galactic latitude. Sources from this sample of 402 sources which did not have a correlated flux density greater than $0.3 \mathrm{Jy}$ on long Earth baselines were excluded from the subset selected for VSOP observations. This selection was accomplished from VLBA observations which are described by Fomalont et al. (2000).

\section{Observations and Results}

The ground array that is scheduled with HALCA for survey observations depends on the source declination. Sources south of $-30^{\circ}$ use Mopra, Hobart, Ceduna and Hartebeesthoek. Observations of more northern sources use Shanghai, Usuda, Kashima or Hartebeesthoek, Noto, Torun, Kalyazin, the Green Bank 140-ft and/or Arecibo. Generally, four ground telescopes are scheduled for each experiment, although usually only three (and sometimes two) telescopes are available for the observations. Most VSOP Survey observations are correlated with the Penticton correlator. A complete discussion of the procedures for reducing the VSOP Survey observations has been given by Moellenbrock et al. (2000). Data are fringe-fit in AIPS and imaged in Difmap.

As of 2000 July, 198 of the 289 selected sources $(69 \%)$ had been observed. In $\sim 10 \%$ of observations no fringes are found to HALCA despite strong fringes being detected on ground baselines, suggesting a timing problem or recording error, and it is planned to reobserve these sources.

One of the main results from the VSOP Survey is the distribution of the normalized visibility amplitude of $\mathrm{AGN}$ at the sub-milliarcsecond level. This is directly related to the average angular size distribution and, hence, the brightness temperature distribution, and will be used as a test of models of superluminal component ejection in AGN which produce the apparent brightness temperatures well in excess of $10^{12} \mathrm{~K}$. The distribution will also indicate which populations of sources can be detected by future space missions (Hirabayashi 2001). In addition, the Survey results, when incorporated with other multiwavelength data, will add to the understanding of many astrophysical phenomena.

\section{References}

Fomalont, E.B. et al. 2000, ApJS in press

Hirabayashi, H., Hirosawa, H. et al. 1998, Science, 281, 1825 (erratum 282, 1995)

Hirabayashi, H., Hirosawa, H. et al. 2000a, PASJ in press

Hirabayashi, H., Fomalont, E.B. et al. 2000b, PASJ in press

Hirabayashi, H. 2001, these proceedings

Moellenbrock G.A., et al. 2000, in Astrophysical Phenomena Revealed by Space VLBI, ed H. Hirabayashi, P.G. Edwards, D.W. Murphy (ISAS, Sagamihara) p177

Murata, Y., Hirabayashi, H., Edwards, P.G. 2001, these proceedings 